\title{
Ethnic difference in lung cancer: an important issue in a globalized society
}

This special Series of the fournal of Thoracic Disease explores the impact of ethnicity and socioeconomic/educational background on the biology, tumorigenesis and treatment outcome of patients with lung cancer. It is designed to provide insights and promote discussion on this issue.

Lung cancer is the most common newly diagnosed malignancy, and the leading cause of cancer deaths worldwide. The disease remains a major global health concern.

It has long been recognized that different ethnicities exhibit distinct patterns of the patient demographics and biology. For the purposes of this special issue, ethnicity is defined as a group with a common ancestry, culture or history.

There is mounting evidence that ethnicity does not just affect lung cancer epidemiology but also the efficacy and toxicities of chemotherapies. We previously published a common arm analysis using arms from a US and a Japanese phase III clinical trial designed prospectively to be identical in schedule and dosing. By removing confounding variables normally inherent to international trial comparisons, we found that Asian patients had a longer survival and higher response rates, yet greater toxicity to chemotherapy than their Caucasian counterparts. We explored potential genotype-related associations with patient outcomes to investigate these differences (Gandara DR, Kawaguchi T, Mack PC, et al. 7 Clin Oncol 2009).

In this Series, along with these results, Dr. Li, Dr. Soo and colleagues observed a similar differential pattern of treatment efficacies and side effects of cytotoxic chemotherapy through a systematic review of publications. Also, given the emergence and significance of immune checkpoint inhibitors, Dr. Lee, Dr. Ahn, et al. focused on the ethnic difference of this new treatment paradigm. Unlike cytotoxic chemotherapy, Asian and Western lung cancer patients had comparable response rates and survival benefits from immune therapy, although with a higher incidence of immune-related adverse events in Japanese patients.

It is accepted that certain single-nucleotide polymorphisms can be associated with treatment efficacy as well as side effects; however, specific tumor mutations can have a more pronounced impact on treatment efficacy, particularly in the era of molecular targeted therapy.

Variations in mutation frequencies across populations may in part be triggered by local environmental factors, including smoking, with evolutionary forces subsequently resulting in dynamic genomic changes

Dr. Izumu clarified the differences in the frequencies of somatic mutations by ethnicity based on two large-scale epidemiological studies from The Cancer Genome Atlas (TCGA) and the Japanese Molecular Epidemiology study (JME). Distinct differences in tumor mutational patterns between Caucasian and Japanese cases are evident, even after considering smoking status. Other environmental factors as well as polymorphisms should be explored to explain these differences more clearly.

A further confounding issue stems from the concept that ethnic differences may be an indirect surrogate marker for access to healthcare and cultural barriers. Dr. Komiya and Dr. Wen, Dr. Guo and colleagues, based on their career experiences, discuss the impact of socioeconomic and educational differences between USA and Japan or China on research and clinical practice.

Given these discussions, ethnicity remains an important and complex characteristic that needs to be recognized, and the understanding should be of great help when it comes to design and conduct of clinical studies, cancer prevention and individualized treatment.

Finally, we are grateful for the opportunity to discuss this important issue and hope that the readers will find the information useful and an inspiration for the further discussion.

\section{Acknowledgments}

Funding: None.

\section{Footnote}

Provenance and peer review: This article was commissioned by the editorial office, Fournal of Thoracic Disease for the series "Ethnic Difference in Lung Cancer". The article did not undergo external peer review. 
Conflicts of Interest: All authors have completed the ICMJE uniform disclosure form (available at http://dx.doi.org/10.21037/ jtd.2020.01.57). The series "Ethnic Difference in Lung Cancer" was commissioned by the editorial office without any funding or sponsorship. TK served as the unpaid Guest Editor of the series and serves as an unpaid editorial board member of Fournal of Thoracic Disease from Dec 2018 to Nov 2020. PCM reports personal fees from Guardant Health, personal fees from Amgen, outside the submitted work.

Etbical Statement: The authors are accountable for all aspects of the work in ensuring that questions related to the accuracy or integrity of any part of the work are appropriately investigated and resolved.

Open Access Statement: This is an Open Access article distributed in accordance with the Creative Commons AttributionNonCommercial-NoDerivs 4.0 International License (CC BY-NC-ND 4.0), which permits the non-commercial replication and distribution of the article with the strict proviso that no changes or edits are made and the original work is properly cited (including links to both the formal publication through the relevant DOI and the license). See: https://creativecommons.org/licenses/by-nc-nd/4.0/.

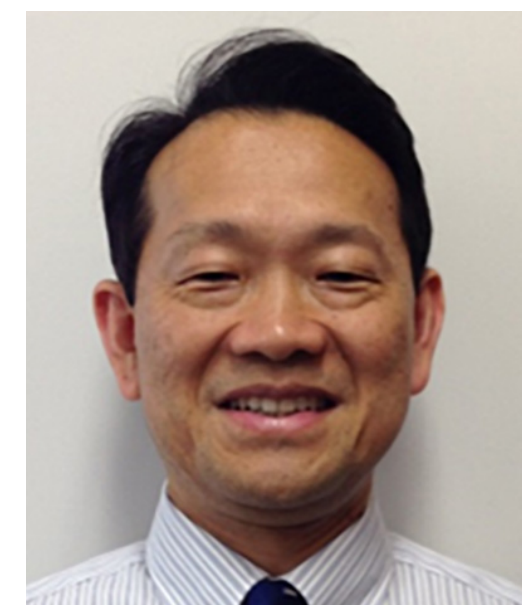

Tomoya Kawaguchi

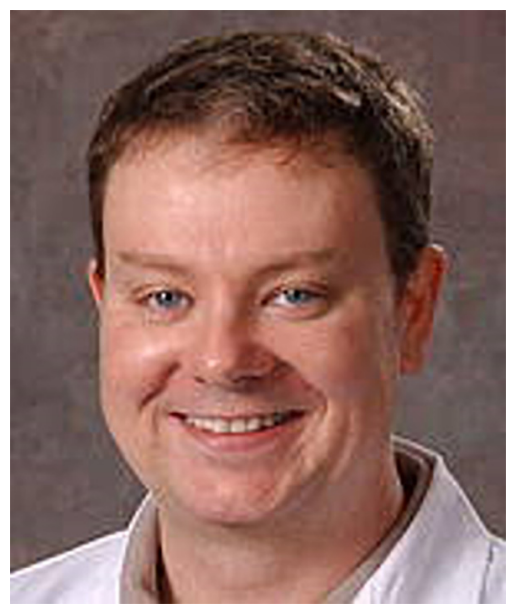

Philip C. Mack

Tomoya Kawaguchi, MD, PhD Department of Respiratory Medicine, Graduate School of Medicine, Osaka City University, Chemotherapy Center, Osaka City University Hospital, Osaka, Japan. (Email: kawaguchi.tomoya@med.osaka-cu.ac.jp)

Philip C. Mack, Adjunct Professor UC Davis Comprehensive Cancer Center, Sacramento, CA, USA. (Email: philip.mack@mssm.edu) Submitted Dec 16, 2019. Accepted for publication Jan 08, 2020. doi: $10.21037 / \mathrm{jtd} .2020 .01 .57$

View this article at: http://dx.doi.org/10.21037/jtd.2020.01.57

Cite this article as: Kawaguchi T, Mack PC. Ethnic difference in lung cancer: an important issue in a globalized society. J Thorac Dis 2020;12(7):3774-3775. doi: $10.21037 /$ jtd.2020.01.57 